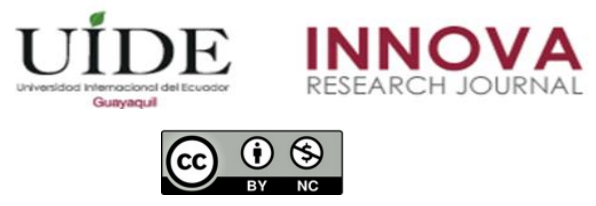

INNOVA Research Journal, ISSN 2477-9024

(Septiembre-Diciembre 2020). Vol. 5, No.3.2 pp. 122-133

DOI: https://doi.org/10.33890/innova.v5.n3.2.2020.1563

URL: http://revistas.uide.edu.ec/index.php/innova/index

Correo: innova@uide.edu.ec

\title{
Los factores que afectan a los emprendimientos en el Ecuador
}

\section{Facts that currently affect entrepreneurship in Ecuador}

\author{
Víctor Hugo Vizueta Tomalá \\ https://orcid.org/0000-0002-1542-9075 \\ Universidad de Guayaquil, Ecuador
}

Autor para correspondencia: victor.vizuetato@ug.edu.ec

Fecha de recepción: 22 de septiembre de 2020 - Fecha de aceptación: 18 de diciembre de 2020

\section{Resumen}

Actualmente los emprendedores se enfrentan a grandes oportunidades, y a la vez, a grandes retos y dificultades, como la degradación del medio ambiente y sus consecuencias en la producción, la crisis económica mundial y local, la proliferación de la administración autocrática de los detentores de poder en las empresas, la protesta social a causa de la insatisfacción política, social y económica del pueblo, bajo nivel de innovación disruptiva que no solo afecta a la producción sino también a la baja calidad de los bienes y servicios que se ofertan. Este artículo tiene como objetivo reconocer los factores que en la actualidad afectan los emprendimientos en el Ecuador, mediante 7 aspectos que afectan a las corporaciones establecidos por Diana Rivenburgh en su libro, The New Corporate Facts of Life. Según los resultados de Global Innovation Index 2019 (Índice de Innovación Global 2019), el Ecuador se ubica en el puesto 99 entre de 129 países y economías de todo el mundo, en desempeño en materia de innovación, esto, contrastado por el crecimiento económico negativo del país, con valores de alrededor de $-7.3 \%$ proyectado por el Fondo Monetario Internacional para el 2020 y a la inestabilidad económica producida por las protestas sociales de octubre de 2019 y los efectos de la crisis sanitaria de la pandemia COVID-19 a nivel global, en la que tienen que ver la globalización, el deterioro del medio ambiente, y la falta de liderazgo empresarial para dar respuesta y soluciones a los retos y oportunidades que se presentan. Palabras claves: emprendimiento; desarrollo; innovación.

\begin{abstract}
Entrepreneurs currently face great opportunities as well as great challenges and difficulties, such as the degradation of the environment and its consequences on production, the global and local economic crisis, the proliferation of the autocratic administration of power holders in the companies, social protest due to the political, social and economic dissatisfaction of the people, low level of disruptive innovation that not only affects production but also the low quality of the goods and services offered. This article aims to recognize the factors that currently affect entrepreneurship in Ecuador, through 7 aspects that affect corporations established by Diana Rivenburgh in her book, The New Corporate Facts of Life. According to the results of the Global Innovation Index 2019 (Global Innovation Index 2019), Ecuador is ranked 99th out of 129
\end{abstract}


countries and economies around the world, in terms of innovation performance, this, this contrasted by economic growth negative for the country, with values of around $-7.3 \%$ projected by the International Monetary Fund for 2020 and to the economic instability produced by the social protests of October 2019 and the effects of the health crisis of the COVID-19 pandemic at a global level, in which globalization, the deterioration of the environment, and the lack of business leadership to provide answers and solutions to the challenges and opportunities that arise have to do.

Keywords: entrepreneurship; development; innovation.

\section{Introducción}

El objetivo de este artículo es analizar los factores que afectan los emprendimientos en el Ecuador, enfocados desde los retos y oportunidades del mundo corporativo descritos por la consultora de negocios Diana Rivenburgh,en su nuevo libro, The New Corporate Facts of Life. Reconocer la problemática que afecta el ecosistema emprendedor en el Ecuador, como lo son: la innovación disruptiva; inestabilidad económica; agitación social; cambios en los depositarios del poder; degradación ambiental; globalización; cambios demográficos, ayudará a los emprendedores a replantar nuevas estrategias y un nuevo modelo de negocio más, que le permita capitalizar y manejar los retos y oportunidades con cautela, de ahí su relevancia.

En este artículo se presentan los temas de estudio mediante una revisión de la literatura donde se analiza y discute informes en el campo teórico, crítico y analítico del ecosistema emprendedor, en cuanto a las causas o factores que afectan su actividad en el Ecuador.

Asimismo, el artículo se estructura de la siguiente forma: en la segunda parte se presentan los 7 factores que afectan a los emprendimientos de acuerdo a los retos y oportunidades descritos Diana Rivenburgh, en su nuevo libro, The New Corporate Facts of Life; en la tercera parte se expone el marco teórico; en la cuarta parte se presentan los resultados basados en una revisión bibliográfica, en la cuarta parte se define la metodología aplicada y para finalizar, en la quinta sección aparecen conclusiones del trabajo investigativo.

\section{Los factores que afectan al emprendimiento}

El Diccionario de la Real Academia de la Lengua Española, define "factor" como "Elemento o causa que actúan junto con otros". El Oxford English and Spanish Dictionary dice que el factor es el "Elemento, circunstancia, influencia, que contribuye a producir un resultado", por ejemplo los factores que determinan la inflación en un país. Según estas definiciones el factor es el productor o generador de resultados que pueden ser positivos o negativos según le caso. Por ejemplo, el factor que determina que el agua llegue a su punto de congelación es la "temperatura" o uno de los factores que afectan la capa de ozono es la quema de combustibles fósiles. Por lo tanto, entendemos por factor a aquellos elementos causantes del cambio, evolución o transformación de los hechos, sucesos o fenómenos que se suscitan en la naturaleza o en el ámbito social, cultural, económico, empresarial etc., y que se obtengan ciertos resultados producto del cambio. 
Diana Rivenburgh (citada por Zwilling, 2013) en su libro, The New Corporate Facts of Life, menciona que los factores que inciden o afectan al mundo de las corporaciones y en consecuencia, al mundo del emprendimiento son: la innovación disruptiva; inestabilidad económica; agitación social; cambios en los depositarios del poder; degradación ambiental; globalización; cambios demográficos.

\section{Innovación disruptiva}

La innovación disruptiva conocida también como "Flee or fight in response to profit", es decir, "Salir o luchar en respuesta al beneficio" (Giner, 2018), se da "cuando se implementan nuevas tecnologías o nuevos modelos de negocio y superan el mercado en el que se desenvuelven; por lo tanto, las empresas se transforman, incluso los sectores industriales" (Corichi, Pérez, Pérez y Valencia, 2018). Los emprendimientos que están fuera de la competencia en el mercado, son aquellos que no han implementado nuevas tecnologías o nuevos modelos de negocio. La carencia de innovación disruptiva afecta no solo los procesos productivos sino la calidad de servicio que se oferta. Como indica (Lettice y Thomond, 2002) "Sin embargo la innovación incrementa la incertidumbre y la presión del mercado".

\section{Inestabilidad económica}

Se define a la inestabilidad o falta de estabilidad, al peligro de que algo cambie. Es así que los factores que determinan la inestabilidad económica en américa latina en las últimas décadas según el Instituto Interamericano para el Desarrollo Económico, Social del Banco Interamericano de Desarrollo (BID), son: factores internos como: la crisis de la inflación cambiaría, crisis bancarias, crisis bancarias sistémicas y crisis de deuda; y, factores externos como: el volumen y los precios del comercio internacional de bienes y de servicios, las remesas que los trabajadores emigrantes hacen a sus familias, la propensión a los desastres naturales en la región, la estructura de la producción y las políticas fiscales, monetarias o financieras.

\section{Agitación social}

Por protesta social, se entiende las diversas y disímiles expresiones de descontento, disgusto, inconformismo, enojo, cólera, desazón o pesadumbre, experimentados por diferentes sectores sociales urbanos frente a situaciones estructurales o coyunturales (Torrejano, 2009). La agitación social desemboca en una recesión económica o época de vacas flacas que se ven reflejadas cuando la tasa de variación del PIB es negativa durante dos trimestres consecutivos, que trae consigo la reducción o disminución del consumo y la inversión, y aumenta el desempleo, factores que afectan los emprendimientos.

\section{Cambios en los depositarios del poder}

Los depositarios del poder o detentadores del poder son aquellos que manejan y controlan los bienes y servicios, además de los factores de la producción y el comercio. De acuerdo a (Beltrán, 2011) "Las organizaciones son, además, sistemas de poder y, por lo tanto, sus objetivos, funciones y roles se encuentran permanentemente en disputa". Los roles establecen quién hace qué, quién decide, quién obedece, y quién controla a quién en una corporación, 
además evidencian los intereses de los depositarios de poder. Por ejemplo, en 1975, el ingeniero de la empresa Kodak, Steve J. Sasson, que inventó el primer modelo de cámara digital en 1975, al conceder una entrevista realizada por el New York Times, mencionó: "Mi prototipo era grande como una tostadora, pero a los técnicos les encantaba, pero era una fotografía sin película, por lo que la reacción de la directiva fue: "Eso está bien, pero no se lo digas a nadie" (Deutsch, 2008), como podemos ver el cambio que requería la empresa Kodak de los rollos de cámara a la cámara digital dependió de la decisión de los detentores del poder o depositarios del poder en este caso de sus directivos. Finalmente, la compañía declaró la quiebra en 2013 ya que acabó por ser superada por las empresas que se adaptaron más rápidamente a la tecnología digital (Giner A, 2018).

\section{Degradación ambiental}

La sostenibilidad medioambiental está seriamente amenazada, pues la emisión de dióxido de carbono se ha acelerado, se pierden bosques, hay sobreexplotación de peces en los mares, especies que van camino a la extinción (ONU, 2013). Las industrias y emprendimientos son los que más contribuyen al deterioro medioambiental por la sobreexplotación de los recursos naturales y la emisión de gases tóxicos a la atmósfera, por lo que, se necesitan negocios o empresas amigables con el ecosistema natural que inviertan en productos, servicios, procesos con esta finalidad. Pero estas acciones producen un sobre costo que el consumidor no está dispuesto a pagar.

\section{Globalización}

James H. Mittelman, profesor de relaciones internacionales e investigador en economía política internacional, considera que "La globalización es una fusión de procesos transnacionales y estructuras domésticas que permiten que la economía, la política, la cultura y la ideología de un país penetre en otro (Morales, s.f.). La globalización ha incrementado las incertidumbres, los riesgos y las oportunidades por la complejidad del entorno en el que se deben desenvolver los negocios actuales. Los emprendedores actuales deben estar preparados para responder rápidamente a las nuevas amenazas y oportunidades que la globalización transmite rápidamente alrededor del mundo. Con toda seguridad podemos afirmar que la globalización está cambiando y dirigiendo la innovación y el emprendimiento.

\section{Cambios demográficos}

El cambio demográfico conocido como un cambio en la composición de la edad en la población se define como: el envejecimiento de la población, la jubilación inminente de los denominados baby boomers (es un término que describe a las personas que nacieron durante la explosión de natalidad en la etapa posterior a la Segunda Guerra mundial 1948-1960) y el descenso de los niveles de natalidad (Foretica, 2010). El escenario futuro de las empresas será un mayor gasto en sueldos y gestión del talento humano, donde los mayores o senior, serán un porcentaje más elevado en las plantillas que los jóvenes, a esto, generará una crisis del talento humano lo que algunos autores han denominado la guerra del talento humano ya que la consideran una amenaza a la productividad e innovación. 


\section{Metodología}

Este trabajo se propone indagar y definir cuáles son los factores que afectan los emprendimientos en el Ecuador, características del contexto que determinan el fracaso de un emprendimiento. Luego de analizar la potencialidad de las diferentes técnicas de análisis, se resolvió aplicar la investigación exploratoria, descriptiva, explicativa y técnicas de recopilación documental que consiste en la recuperación, análisis, crítica e interpretación de datos secundarios. La revisión de las fuentes y la obtención de información documental en el proceso de investigación tiene como finalidades: identificar nuevos problemas; conocer el estado de la cuestión en nuestro tema; conocer el alcance y relevancia del problema dentro de nuestra comunidad científica; conocer los posibles enfoques de la investigación y adoptar uno de modo coherente; obtener información para el desarrollo de la investigación misma; confrontar nuestras conclusiones con la investigación previa (Gómez, 2010). Los datos analizados provienen de la revisión bibliográfica y de fuentes como el Instituto de Estadísticas y Censos (INEC), El Banco Central del Ecuador, El Fondo Monetario Internacional (F.M.I.), Global Innovation Index 2019, Banco Mundial con el apoyo de Banco Central del Ecuador.

\section{Resultados}

A partir del 2015, el promedio de crecimiento del PIB trimestral en Ecuador ha sido de $0 \%$, a consecuencia por el desplome del precio de barril de petróleo en alrededor de $50 \%$ a partir del 2014, la falta de ahorro fiscal durante la época de bonanza petrolera, la baja inversión extranjera directa, y el creciente déficit fiscal (Lasio, Amaya, Zambrano, Ordeñana X, 2020). El Banco Central del Ecuador, proyectaba para el 2020 un crecimiento del $0.7 \%$ pero por efecto de la crisis global de la pandemia COVID-19, el crecimiento económico del país han cambiado drásticamente hacia el lado negativo, con valores de alrededor de $-7.3 \%$ proyectado por el Fondo Monetario Internacional para el 2020. La crisis económica y la emergencia sanitaria ha generado diversos escenarios y problemas para la actividad empresarial, siendo las más afectadas el sector de la transportación, el comercio, hotelero, turismo, entretenimiento, la industria textil, y en menos grado el sector de los emprendimientos tecnológicos, eso conlleva a la caída de las ventas, crecimiento del desempleo y la disminución de los puestos de trabajo. 


\section{Figura 1}

Estimación de los Empleos en riesgo en 2020

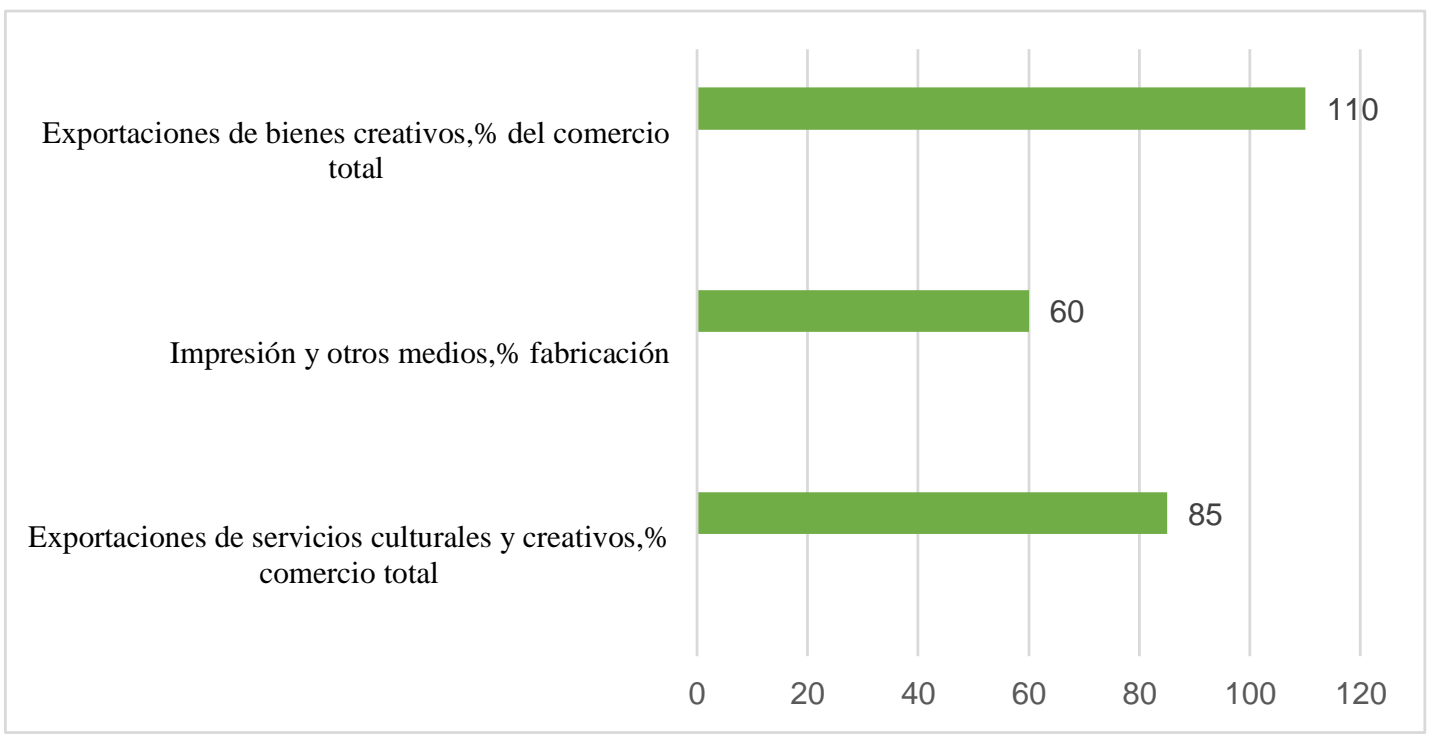

Fuente: Cámara de Industrias y Producción

Según los resultados de Global Innovation Index 2019 (Índice de Innovación Global 2019), que clasifica y desglosa el desempeño en materia de innovación de 129 países y economías de todo el mundo, el Ecuador en el 2018 se ubica en el puesto 98 y en el 2019 en el puesto 99, por lo que existe una evolución negativa del pilar 'Sofisticación de los negocios', que contiene sub-pilares importantes como la vinculación entre la universidad y la industria y la capacitación formal que las empresas están proporcionando a sus colaboradores. Esto ha dado como resultado que Ecuador se encuentre muy por detrás de otros países (Amaya, 2019). Estos indicadores demuestran que la innovación en el mundo empresarial es de mucha importancia. Por lo tanto, que los emprendimientos en el Ecuador no sean superados y tengan una ventaja competitiva crucial en el futuro deben de crear nuevas formas de hacer los productos. 


\section{Figura 2}

Evolución del índice global de innovación de Ecuador

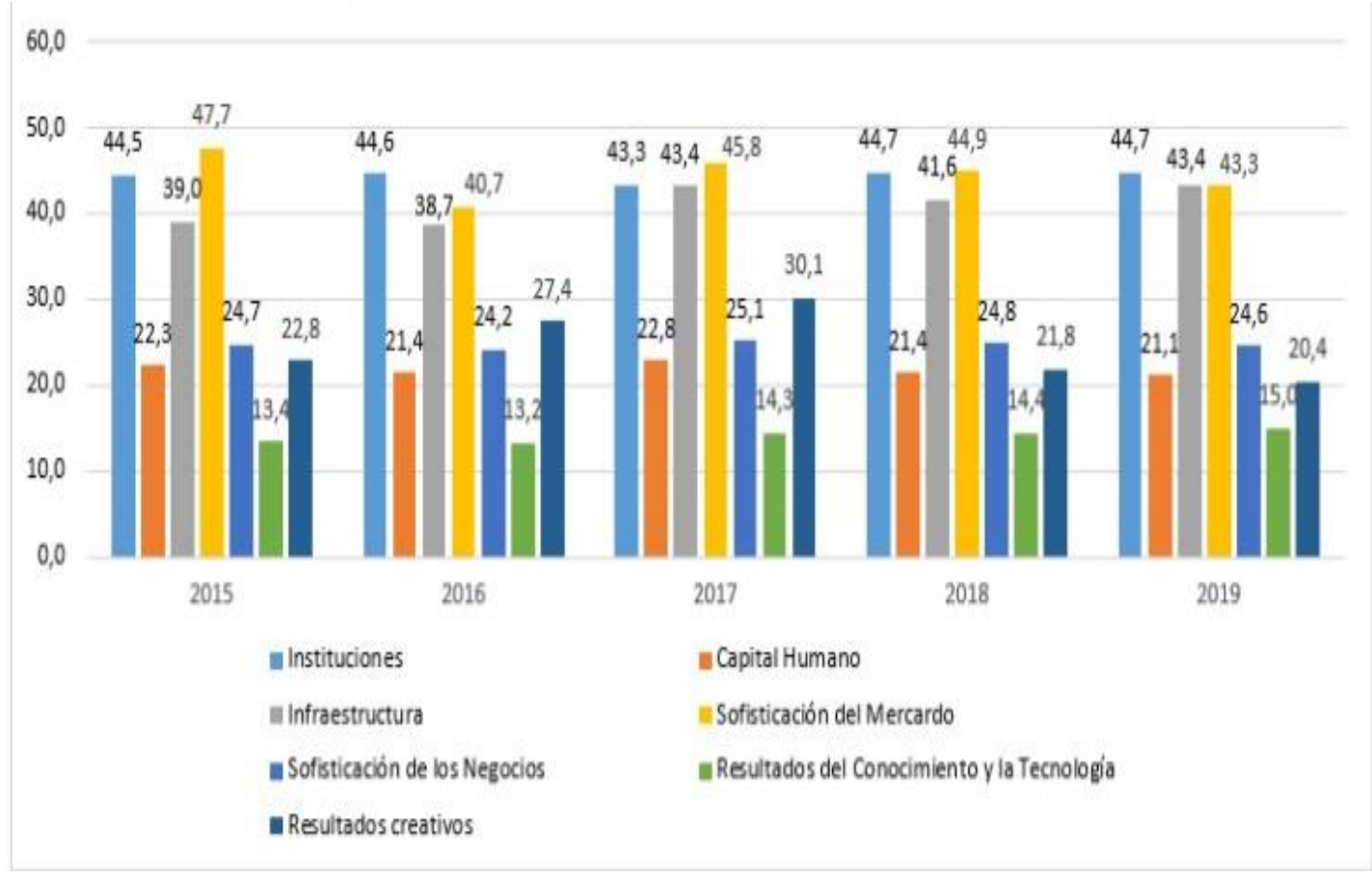

Fuente: Amaya A. (2019). Resultados Global Innovation Index 2019: "Ecuador baja en innovación, se presentan obstáculos para avanzar". Recuperado de http://www.desdelcentro.org/resultados-global-innovation-index-2019ecuador-baja-innovacion-se-presentan-obstaculos-avanzar

Las manifestaciones sociales del último trimestre del 2019 en el Ecuador, llevaron al presidente de Ecuador, Lenín Moreno a recalibrar el programa económico y suprimir el decreto 883, con el que se eliminaban los subsidios a la gasolina extra y al diésel. La medida estaba destinada a bajar el déficit fiscal para 2020 y a reducir el gasto por lo menos USD 1.500 millones anuales en momentos en que Ecuador ha tenido que optar por un plan de austeridad. La agitación social vivida en octubre de 2019 en el Ecuador, trajo como consecuencia la disminución de las ventas y el comercio, además, el sector turístico se vio afectado y las conversaciones de inversión extranjera en el sector agrícola y agroindustrial de la Sierra centro, se suspendieron. la evaluación de daño y pérdidas afectaciones por paro nacional del 3 al 14 de octubre de 2019 república de Ecuador, realizada por Banco Mundial con el apoyo de Banco Central del Ecuador, determinaron que el valor de las afectaciones derivadas de la movilización ascendió a USD 821,68 millones como se muestra en el siguiente grafico por sectores. 


\section{Figura 3}

Estimación de afectaciones en todos los sectores y sub - sectores

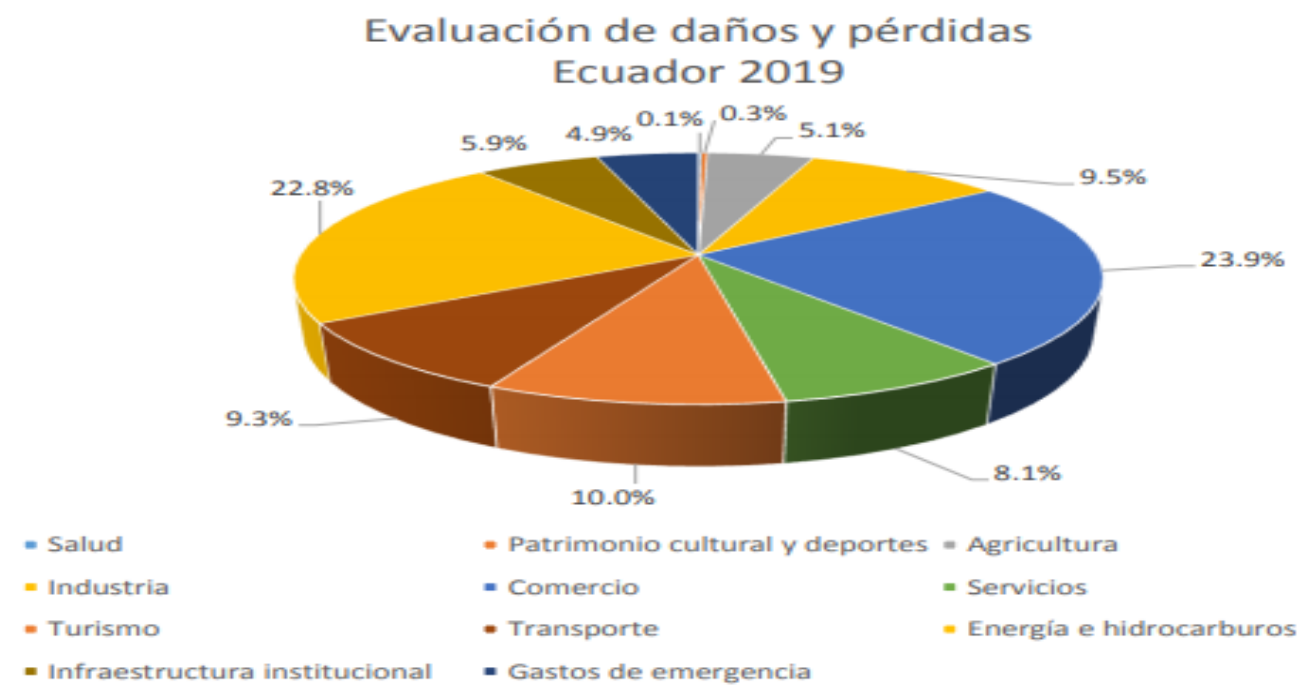

Fuente: Banco Central del Ecuador, 2019

En emprendimiento, nociones como tomar riesgos y no tener miedo al fracaso son cruciales ya que determinarán el proceso de toma de decisiones de un emprendedor, ya sea decisiones de inversión o incluso emprender nuevamente en el caso de haber fallado una o varias veces (AEI, 2013). Los cambios en los depositarios del poder tienen relación al estilo de liderazgo que fueron definidos y clasificados por Lewin en 1939, en liderazgo autocrático, democrático y liberal o consultivo conocido también Estilo Laissez Faire. En relación con las 23 provincias de la República del Ecuador, podemos decir que también son administradas en su mayoría con un estilo de Liderazgo Autocrático (Pazmiño, Beltrán y Gallardo, 2016).

\section{Tabla 1}

Los tipos de Liderazgo

\begin{tabular}{ll}
\hline Tipo de Liderazgo & Decisiones \\
\hline Autocrático & $\begin{array}{l}\text { Se toman decisiones de forma independiente con poco o ningún aporte desde } \\
\text { el resto del grupo }\end{array}$ \\
\hline Democrático & $\begin{array}{l}\text { Fomenta la participación del equipo en la toma de decisiones, delega la } \\
\text { autoridad e involucra a los subordinados para decidir métodos; además, } \\
\text { retroalimenta y guía. }\end{array}$ \\
\hline Liberal O Consultivo & $\begin{array}{l}\text { Los líderes consultivos ofrecen poca o ninguna orientación a sus } \\
\text { colaboradores, además de dejar el proceso de toma de decisiones en manos } \\
\text { Estilo Laissez Faire grupo sin orientación. Sin embargo, este estilo podría ser eficaz siempre } \\
\text { y cuando los miembros del grupo sean altamente calificados y especializados } \\
\text { en el área de su trabajo. }\end{array}$ \\
\hline
\end{tabular}

Elaborado por el autor.

Fuente: Pazmiño G., Beltrán M. y Gallardo W. (2016). 
La globalización es un sistema que ha traído para los países en desarrollo el constante incremento de los niveles de desempleo, pobreza y marginalidad que son factores que afectan a los emprendimientos. La globalización es un sistema totalitario que entre otros males ha traído al Ecuador la integración internacional y la desintegración nacional, la internacionalización de la pobreza, la falta de identidad o desterritorialización, la pérdida de las fronteras en las distintas dimensiones de la economía, la información, la ecología, la técnica, los conflictos transculturales y la sociedad civil. El proceso de globalización trae como consecuencias:

\section{Tabla 2}

Las consecuencias de la globalización

\begin{tabular}{ll} 
Destrucción de la Economía & \multicolumn{1}{c}{ Pues si la economía es la acción humana destinada a } \\
establecer las bases materiales de la vida física y cultural \\
de todos los seres humanos en el mundo entero, la \\
globalización es el sistema más ineficaz de la historia \\
humana
\end{tabular}

Destrucción de la naturaleza Tanto en el clima como el agotamiento de recursos naturaleza.

Esto es extensión de las relaciones directas e indirectas

\section{Destrucción social}

\section{Destrucción cultural}

\section{Destrucción política}

Tabla elaborada por el autor. Fuente: García F. (2005).

A lo largo de la historia del Ecuador, el desarrollo nacional ha estado configurado por la explotación de los recursos naturales, este modelo extractivista ha causado problemas en los ámbitos socio económico, socio cultural y en los recursos naturales (aire, agua, flora, fauna, suelo, clima, energía, recursos humanos) que se han visto afectados. En el ámbito socio económico la afectación es notoria en los sectores turísticos, en la economía de las comunidades indígenas y en la disminución de las fuentes de empleos, se suma a esto la pérdida en recursos para procesamiento de la industria químico farmacéutica y la pérdida de conocimiento ancestral sobre propiedades de plantas. Con la Constitución del 2008, se propone el modelo de desarrollo denominado "El buen vivir o Sumak Kawsay", modelo de desarrollo basado en la visión indígena del bienestar, y por las tendencias del desarrollo sustentable, donde el ambiente y la naturaleza pasan a tener un papel protagónico por primera vez en la historia de nuestro país (Puentestar, 2015). Esta nueva visión, o política emergente se alzó como un modelo de desarrollo a largo plazo pero con inconsistencia en su aplicación en temas altamente sensibles como las

Esta obra se comparte bajo la licencia Creative Common Atribución-No Comercial 4.0 International (CC BY-NC 4.0) Revista de la Universidad Internacional del Ecuador. URL: https://www.uide.edu.ec/ 
concesiones petroleras y mineras dentro de áreas protegidas como los páramos andinos o la Amazonía. Se agrega también a esta problemática el bajo porcentaje de inversión de las empresas en protección ambiental que se muestran en la Encuesta de Gasto e inversión privada en Protección Ambiental, realizada por INEC en el 2010, donde se establece que el 32,7\% de las empresas realizan inversiones en protección ambiental en la adquisición de equipos e instalaciones para reducir las emisiones de contaminación.

\section{Tabla 3}

Inversión ambiental de la empresa en el Ecuador

\begin{tabular}{cllll}
\hline \multirow{2}{*}{ Tipo de Empresa } & \multicolumn{3}{l}{ Tiene gasto o inversión ambiental } \\
\cline { 3 - 5 } & Pequeña empresa & $25,5 \%$ & No & Total \\
\hline \multirow{3}{*}{ Porcentaje } & Mediana empresa & $52,5 \%$ & $47,5 \%$ & $100 \%$ \\
& Grande empresa & $69,2 \%$ & $30,8 \%$ & $100 \%$ \\
& Total & $32,7 \%$ & $67,3 \%$ & $100 \%$ \\
\hline
\end{tabular}

Fuente: INEC - Encuesta del Gasto e Inversión Privada en Protección Ambiental (2010)

En transición demográfica hay un periodo en el que la proporción de personas en edades económicamente activas crece de manera sostenida en relación a la de personas en edades dependientes, particularmente de niños (Saad, Murad, Miller y Martínez, 2009). Al aumento de la población en edades económicamente activas, podrá apoyar el despegue económico y contribuir a mitigar la carga económica de personas en edades dependientes, se lo conoce como "bono demográfico", que implica un impacto económico de los cambios geográficos en sectores como la salud, educación, empleo, sistema de pensiones por el efecto del envejecimiento de la población que es un desafío. La transición demográfica en América Latina es muy heterogénea por lo cual la Cepal (2008) clasificó a los países latinoamericanos en cuatro etapas de transición demográfica (muy avanzada, avanzada, plena y moderada) de acuerdo a sus niveles de fecundidad y esperanza de vida. En la fase de transición está ubicado el Ecuador, que había logrado un considerable descenso de la fecundidad a comienzos de los años ochenta pero que en la actualidad está ubicado en un nivel de fecundidad intermedio dentro de la región. El fenómeno vejez en la transición demográfica aumentara la carga financiera a los contribuyentes en Ecuador y en consecuencia afectara los emprendimientos.

\section{Conclusiones}

La innovación disruptiva; inestabilidad económica; agitación social; cambios en los depositarios del poder; degradación ambiental; globalización; cambios demográficos son factores que afectan los emprendimiento a nivel global. El ecosistema emprendedor en el Ecuador está amenazado por los siguientes factores:

- La insipiente implementación de nuevas tecnologías o nuevos modelos de negocio. 
- La crisis de la inflación - cambiaria, crisis bancarias, crisis bancarias sistémicas y crisis de deuda, el volumen y los precios del comercio internacional de bienes y de servicios, las remesas que los trabajadores emigrantes hacen a sus familias, la propensión a los desastres naturales en la región, la estructura de la producción y las políticas fiscales, monetarias o financieras.

- La agitación social que trae consigo la reducción o disminución del consumo y la inversión, y el aumenta el desempleo, factores que afectan los emprendimientos.

- La incipiente inversión del estado y las empresas en productos, servicios y procesos que protejan la sostenibilidad medioambiental.

- El proceso de globalización que incide negativamente en la sociedad, política, economía, naturaleza, salud y cultura de los pueblos.

- La mayor carga financiera a los contribuyentes por el aumento del bono demográfico a causa del fenómeno de la vejez.

- La administración de las empresas al estilo de liderazgo autocrático, que fomenta las decisiones de los depositarios del poder de forma independiente con poco o ningún aporte desde el resto del grupo.

La protección del medio ambiente, la tranquilidad social, el control de transición demográfica, la innovación disruptiva, la economía saludable de un país, las sabias decisiones de los detentores del poder en las empresas, la oportuna respuesta a las amenazas y oportunidades de la globalización son acciones que favorecerán los emprendimientos en el Ecuador.

\section{Referencias bibliográficas}

Aei (2014). El ecuador un país emprendedor en innovador en el 2020. Estrategias para desarrollar un ecosistema de emprendimiento e innovación. Recuperado de https://unctad.org/en/PublicationsLibrary/epf_npd02_Ecuador_es.pdf

Alianza para el Emprendimiento e Innovación (AEI), (2013), Ecuador un país emprendedor e innovador en $2020 . \quad$ Recuperado de https://unctad.org/en/PublicationsLibrary/epf_npd02_Ecuador_es.pdf

Banco Mundial con el Apoyo de Banco Central del Ecuador (2019). Evaluación de daño y pérdidas afectaciones por paro nacional del 3 al 14 de octubre de 2019 república de Ecuador. Recuperado de https://www.bce.fin.ec/images/BANCO_C_ECUADOR/PDF/InformeBMImpactoParoNa cional.pdf

Cepal (2008). Panorama social: América Latina. Recuperado de https://repositorio.cepal.org/bitstream/handle/11362/1229/1/S0800829_es.pdf

Corichi A., Pérez J.C., Pérez J.A. y Valencia K. (2018), Introducción a la innovación disruptiva y su efecto en algunos sectores industriales. Recuperado de file:///C:/Users/User/Downloads/39-149-1-PB.pdf.

Deutsch, C. (2 de Mayo de 2008). At Kodak, Some Old Things Are New Again. The New York Times. Obtenido de https://www.nytimes.com/2008/05/02/technology/02kodak.html

Foretica, (2010). El Cambio Demográfico. El reto del mundo desarrollado. Implicaciones, riesgos y recomendaciones. Recuperado de 
https://www.fundacionseres.org/lists/informes/attachments/1054/el\%20cambio\%20demo gr\%c3\%alfico.pdf.

Giner A. (2018). El modelo de la innovación disruptiva: Estudio de caso de Netflix. Recuperado de $\quad$ https://riunet.upv.es/bitstream/handle/10251/110652/Giner\%20\%20E1\%20modelo\%20de\%20la\%20innovaci\%C3\%B3n\%20disruptiva\%3A\%20Estudio $\% 20 \mathrm{de} \% 20$ caso $\% 20 \mathrm{de} \% 20 \mathrm{Netflix} . p d f ?$ sequence $=1 \&$ isAllowed $=\mathrm{y}$

Gómez J. (2010). La recopilación documental. Para qué y cómo documentarse en Ciencias de la Información Documental. Recuperado de https://digitum.um.es/digitum/bitstream/10201/47175/1/recopilaciondocumentalgomez.pd $\underline{\mathrm{f}}$

Inec (2014). Encuesta del Gasto e Inversión Privada en Protección Ambiental 2010. Recuperado de https://anda.inec.gob.ec/anda/index.php/catalog/340/related_materials

Lettice, F. \& Thomond, P. (2002), "Disruptive innovation explored", paper presented at Ninth IPSE International Conference on Concurrent Engineering: Research and Applications (CE2002). Recuperado de http://www.insightcentre.co.uk/resources/DIExploredCEConf2002final.pdf

Morales F. (s.f.). Globalización: Conceptos, Características y Contradicciones. Recuperado de file:///C:/Users/User/Downloads/Dialnet-Globalizacion-4796216.pdf.

ONU. (2013). Objetivos de Desarrollo del Milenio. Naciones Unidas. Recuperado de http://www.un.org/es/millenniumgoals/pdf/mdg-report-2013-spanish.pdf

Pazmiño G., Beltrán M. y Gallardo W. (2016). Los estilos de liderazgo y su influencia en el desarrollo empresarial: caso pymes de la provincia de Tungurahua - Ecuador. Recuperado de file:///C:/Users/User/Downloads/45-Texto\%20del\%20art\%C3\%ADculo-326-1-1020171211.pdf

Puentestar W. (2015). "La problemática ambiental y el deterioro de los recursos naturales en el ecuador. Una perspectiva desde la geografía”. Recuperado de http://repositorio.puce.edu.ec/bitstream/handle/22000/8665/LA\%20PROBLEM\%C3\%81 TICA\%20AMBIENTAL\%20Y\%20EL\%20DETERIORO\%20DE\%20R.N.\%20EN\%20E L\%20ECUADOR.\%20UNA\%20PERSPECTIVA\%20DESDE\%20LA\%20GEOGRAFI.p df?sequence $=1 \&$ is Allowed $=y$

Rodríguez D. (2016). Emprendimiento sostenible, significado y dimensiones. Recuperado de file://C:/Users/User/Downloads/DialnetEmprendimientoSostenibleSignificadoYDimensiones-5850542.pdf.

Saad, Murad P., Miller T. y Martínez C. (2009). Impacto de los cambios demográficos en las demandas sectoriales en América. Recuperado de https://www.scielo.br/scielo.php?pid=S0102$\underline{30982009000200006 \& \text { script }=\text { sci_abstract\&tlng=es }}$

Torrejano R, (2009). Algo más que piedra y consignas Agitación social vista desde la prensa durante el gobierno de la concentración nacional (1930 - 1934). Recuperado de http://www.scielo.org.co/scielo.php?script=sci_arttext\&pid=S1657-89532009000100008. 